\title{
La investigación sobre comunidades gitanas en España en el contexto europeo: un análisis documental ${ }^{1}$
}

Research on Roma communities in Spain in the European context: a documentary analysis

Francisco Eduardo Haz Gómez

Universidad de Murcia

francisco.haz@um.es

Inés Andreu Ferrer

Universidad de Murcia

ines.andreu@um.es

Manuel Hernández Pedreño

Universidad de Murcia

manuel@um.es

Catalina Guerrero Romera

Universidad de Murcia

cgromera@um.es

Eduardo Romero Sánchez

Universidad de Murcia

eromero@um.es

María Dolores Abellán Giménez

Universidad de Murcia

md.abellangimenez@um.es

\section{Resumen}

El Pueblo Gitano ha estado presente en la historia de Europa y de España, siendo habitual las referencias a él como colectivo vulnerable. Sin embargo, apenas se tiene conocimiento de su situación social desde un enfoque global o multidimensional, es decir, teniendo en cuenta su riesgo social en diferentes ámbitos, como empleo, formación, ingresos, vivienda, salud, relaciones socio-familiares o participación. Mediante el análisis documental o bibliográfico,

${ }^{1}$ Recibido: 30/04/2019 Evaluado: 02/05/2019 Aceptado: 05/05/2019 
este trabajo pretende avanzar en esta línea, realizando una revisión sistemática de la situación de la investigación sobre este colectivo en el contexto europeo, al tiempo que se profundiza en la información que aportan los estudios en España sobre su situación social multidimensional. Los resultados obtenidos desvelan que mientras en Europa el interés científico se centra en su integración, destacando los estudios sobre participación ciudadana, en España priman los estudios que abordan dimensiones relacionadas con sus procesos de inclusión/exclusión, especialmente el de la educación.

Palabras clave: revisión bibliográfica; comunidades gitanas; exclusión social; enfoque multidimensional; España; Europa.

\begin{abstract}
Roma people have been present for centuries in the history of Europe and Spain; being usual references to them as a vulnerable community. Nevertheless, there is barely any knowledge about their social situation from a holistic or multidimensional approach. It means, taking into account their social risk at different areas such as: employment, training, income, housing, health, social and family relations or participation. This article aims to improve the knowledge of Roma community through the documentary or bibliographic analysis in two different ways. On the one hand, a research systematic review is implemented in the European framework. And on the other hand, research is conducted through multidimensional approach to different studies deepening the understanding of social situation of Roma in Spain. The outcomes reveal that in Europe scientific interest is focused on their social integration; studies on participation are highlighted. However, in Spain several researches show that prevailing dimensions are related to their Social Inclusion / Exclusion processes; mainly about Education.
\end{abstract}

Keywords:Bibliographic Review; Roma communities; Social Exclusion; Multidimensional approach, Spain; Europe.

\title{
Introducción
}

A pesar de su llegada a la Península Ibérica hace más de 600 año, antes de que España se configurara como Estado (Vilar, Vilar y Caselles, 2009), y de haber sido la minoría étnica más numerosa en España hasta la llegada de la inmigración masiva en los años noventa del siglo XX; el estudio del pueblo gitano sigue siendo una asignatura pendiente para las ciencias sociales. Su cultura, el aislamiento o sus pautas residenciales pueden ser causa de ello. Laparra (2007) plantea dos hipótesis sobre las posibles explicaciones de la carencia de estudios sobre este grupo. Por un lado, falta de interés por parte de los organismos públicos y de la comunidad científica, acentuada por un mecanismo defensivo desde el colectivo gitano, que recela de ser objeto de estudio o atención. Por otro lado, a raíz de la legislación vigente, y en pos de evitar una posible discriminación, se impide realizar censos sobre perfiles sensibles a la categorización, entre los que se incluye el origen étnico. De ahí que obtener cifras reales a nivel estatal sobre este colectivo sea una tarea casi imposible. Este obstáculo disuade a muchos científicos de realizar análisis demográficos a nivel macrosocial. Estas lagunas de conocimiento impiden que las políticas sociales que intervienen sobre estas comunidades tengan un diseño adecuado a su realidad, porque no se llega a 
conocer la dimensión social del pueblo gitano, su distribución demográfica o sus características sociales generales, que sin duda son plurales y complejas.

Los procesos de inclusión/exclusión social se conforman en torno a la acumulación de ventajas/desventajas sociales en una serie de ámbitos. En España las dimensiones de la exclusión social se vienen definiendo como siete dimensiones en las que se puede medir el grado de riesgo social de todo ciudadano o colectivo (Hernández Pedreño, 2010). Aunque estos ámbitos o dimensiones poseen diferentes denominaciones, existe gran consenso en concretarlas en siete: ingresos, trabajo, vivienda, salud, educación, relaciones sociofamiliares y participación. Estos ámbitos vitales mantienen su correspondencia con las diversas líneas de intervención social. Es decir, la dimensión ingresos se correspondería con la política social de ingresos o de rentas, la de trabajo con la política de empleo, y así sucesivamente. El Observatorio de la Exclusión Social de la Universidad de Murcia lleva más de una década trabajando con un modelo metodológico que permite esta aproximación multidimensional al estudio de los procesos de inclusión/exclusión social, así como a la respuesta institucional en cada uno de ellos, aplicándolo recientemente a la investigación de diversos colectivos, como personas sin hogar (Hernández Pedreño, 2016) o jóvenes (Hernández Pedreño, 2018), entre otros.

Desde el año 2016 esta entidad ha conformado un grupo de investigación-acción sobre el estudio de las comunidades gitanas. Se adopta esta perspectiva de investigación- porque se pretende, no solo estudiar a las comunidades gitanas de un modo científico, sino aportar su visión a través de diferentes instituciones que las representan -y los técnicos que con ellas intervienen-, haciéndoles así partícipes de su propio autoconocimiento. De este modo, se aplica el principio antropológico emic-etic (visión propia y visión ajena) al mismo tiempo que se trabaja con el modelo teórico de exclusión social multidimensional, que será sumamente útil para el futuro diseño de políticas de inclusión.

Bajo este modelo de análisis multidimensional de la exclusión social y a partir de la revisión documental este trabajo pretende realizar una primera aproximación a situación del colectivo gitano en España, si bien previamente se contextualizará en la investigación científica que se desarrolla en Europa y las principales áreas de interés. Este fin se alcanzará a partir de dos tipos de revisión bibliográfica o documental: la revisión sistemática, para el contexto europeo y la exhaustiva para España.

A pesar de las limitaciones de ambas aproximaciones, este trabajo ofrece una visión bastante inédita, tanto sobre el interés científico por las comunidades gitanas en Europa, como de su situación social desde un enfoque multidimensional en España.

\section{Objetivos y metodología}

Como ya se ha anunciado, este trabajo parte de una finalidad clara que se plantea como objetivo general: analizar la situación de la investigación sobre las comunidades gitanas en relación a sus procesos de exclusión/inclusión social multidimensionales, tanto a nivel europeo como español. Las limitaciones metodológicas de tiempo y recursos permitirán alcanzar este objetivo, si bien, adecuado a dos objetivos específicos.

\section{Objetivos}

No 7, 2019. Página | 119 
Respecto a la situación de la investigación sobre comunidades gitanas en Europa, el primer objetivo específico se centrará en ofrecer una primera visión que permita caracterizar cuantitativamente el tipo de investigación existente, a partir de las disciplinas que lo investigan, los ámbitos que contemplan o la metodología empleada, entre otros.

El segundo objetivo específico tiene un mayor calado cualitativo, pues pretende profundizar en la situación social de las comunidades gitanas en España aplicando el enfoque multidimensional, es decir, ahondar en la información que proporcionan los estudios realizados en España sobre cada una de las dimensiones contempladas.

\section{Metodología}

Como se ha visto, el marco teórico de este trabajo e hilo conductor entre ambos objetivos es el concepto multidimensional de la exclusión social, no obstante, el alcance de dichos fines se realizará con diferentes técnicas de análisis documental. En particular el análisis europeo, más cuantitativo, se lleva a cabo mediante la revisión sistemática; mientras el análisis español, más profundo y cualitativo, se hará a través de la revisión bibliográfica, si no claramente exhaustiva, si bastante extensa.

En ambos tipos de análisis existen precedentes metodológicos aplicados a otros colectivos, aunque aplicados al colectivo gitano también se cuenta con el estudio realizado por Ferrer (2003) mediante revisión bibliográfica, el que se da cuenta de la situación de la investigación en la dimensión salud.

En nuestro caso, el diseño de la investigación comienza con la elaboración de un protocolo de revisión cuya finalidad es la especificación de una serie de criterios de selección de aquellos trabajos que se van a examinar, teniendo en cuenta las diferentes preguntas iniciales de investigación en relación a los objetivos perseguidos. Se plantearon seis cuestiones:

1. ¿Qué dimensiones de análisis de la exclusión se tratan en las investigaciones?

2. ¿Desde qué disciplina de las ciencias sociales se realiza?

3. ¿De qué tipo de documento se trata? (artículo, libro, tesis doctoral, etc.)

4. ¿A qué contexto geográfico se circunscribe?

5. ¿Qué metodología siguen estos trabajos?

6. ¿A qué fecha/periodo de publicación/análisis se hace referencia?

Estas preguntas formuladas han servido para establecer seis categorías analíticas comunes a ambos tipos de análisis (sistemático en Europa y exhaustivo en España).

Por otra parte, se implementó la estrategia de búsqueda que comprende dos elementos. El primero de ellos es la determinación de las fuentes a consultar. Y el segundo, las palabras clave que se utilizan en los motores de búsqueda de las fuentes seleccionadas.

Asimismo, dado el volumen de documentación encontrada en una primera búsqueda se ha optado por diferenciar los periodos de estudio para los diferentes análisis. En el caso de la revisión sistemática, aplicada al marco europeo, se estudiarán los documentos comprendidos entre los años 2007 y 2017. Por otra parte, con el fin de enriquecer la descripción, se amplía a las últimas décadas el análisis exhaustivo sobre la situación social de las comunidades gitanas en España.

De forma más detallada y específica se explica a continuación el protocolo seguido en cada tipo de análisis, así como las decisiones muestrales aplicadas. 
Revisión sistemática de la investigación en Europa.

Para la realización del primer objetivo específico se aplica la revisión sistemática de estudios referida al marco europeo. El diseño de la metodología de análisis se estructura en cuatro apartados (ámbito geográfico, fuentes documentales, términos clave para la búsqueda y periodo a analizar).

El criterio para delimitar el ámbito geográfico ha sido abarcar toda Europa, tanto países, como entidades supranacionales, como la unión europea o regiones dentro de este continente, como Europa del Este.

Respecto al idioma y las palabras clave, dada la pluralidad de lenguas y estudios, se han seleccionado aquellos estudios en lengua inglesa, y por ende, los términos de búsqueda se encuentran en este idioma. Esto responde a dos motivos: el primero obedece a que lengua inglesa es la estándar u oficial en las publicaciones a nivel internacional; y el segundo, por evitar redundancias con otras publicaciones que pudieran estar en español. Los términos o palabras clave de búsqueda fueron dos: Gypsy y Roma, puesto que estos designan de manera general a los integrantes de la etnia gitana.

Las fuentes documentales utilizadas en la revisión sistemática son principales bases de datos que recogen la mayoría de la producción científica del objeto de estudio: Scopus, ISI Web of Science, y ProQuest. De estas bases de datos se han extraído las investigaciones con mayor índice de impacto, obteniendo 100 muestras o documentos.

Respecto a la selección del periodo de análisis, éste se ha fijada en 2007-2017. Esta temporalidad se justifica por varios aspectos, sociales y metodológicos. Por un lado, estaría el volumen de documentación existente para periodos más extensos; por otro, la coincidencia del periodo con el inicio y final de la crisis económica, al tiempo que 2007 es el año en el que los países de la Unión Europea comienzan a implantar los planes de inclusión social de las comunidades gitanas.

Tras aplicar los anteriores criterios de búsqueda se obtuvieron los siguientes resultados para cada base de datos. En Scopus se obtuvieron 1.461 resultados, en Web of Science 382 y, por último, en ProQuest 1.171 documentos. Tras una depuración de los datos según el impacto, se seleccionaron las 100 primeras entradas con el mayor nivel, sobre las cuales se verán más adelante los resultados obtenidos.

Revisión exhaustiva de la investigación en España.

El diseño para la realización del segundo objetivo específico, el análisis de la situación social de las comunidades gitanas en España, se lleva a cabo a través de una metodológica de revisión exhaustiva de aquellos trabajos realizados sobre este colectivo en España. Como en la revisión sistemática llevada a cabo en referencia al marco europeo, esta también comparte las mismas categorías analíticas (ámbito geográfico, fuentes documentales, términos clave para la búsqueda y periodo a analizar). Sin embargo, siguiendo el propósito de este segundo objetivo se profundiza muchos más en la información contenida en los estudios.

El ámbito geográfico está circunscrito a todo el territorio español. Incluyendo los tres niveles de desagregación territorial (nivel estatal, autonómico y local). 
Siguiendo un criterio de unidad y coherencia en la metodología de ambos objetivos específicos los términos de búsqueda escogidos son: gitano y romaní. Los mismos términos en español que los utilizados en inglés.

En lo que respecta a las fuentes documentales, en lugar de acudir a bases de datos se optó por realizar la búsqueda siguiendo varios criterios. Inicialmente, los recursos digitales para la búsqueda se realizaron a través de Researchgate, Google Académico y Dialnet. Dada la limitada exhaustividad que aseguraba este primer criterio y el escaso número de documentos obtenido, se amplió la búsqueda por otras vías. Por un lado, se seleccionaron todos los documentos que figuraban en el Catálogo Alba de la Universidad de Murcia, que da cuenta de los fondos bibliotecarios de ésta. Por último, se recurrió al examen en profundidad de las referencias y bibliografía, ampliándose así de nuevo la muestra.

No obstante, no fue posible aplicar el análisis en profundidad a todos los documentos encontrados, ya que a algunos de ellos no fue posible acceder, bien por no estar en abierto en la red, o bien por no estar en los fondos documentales de las bibliotecas de la Universidad de Murcia. El volumen total de los textos analizados es de 62 documentos de diversos tipos (artículos, informes, libros y capítulos, tesis doctorales y ponencias.

Por último, en relación al periodo que abarca esta revisión exhaustiva, el rango de años se extendió desde 1978 a 2017. La ampliación de este periodo de estudio respecto al análisis sistemático se justifica en gran medida por el escaso número de documentos existentes, sobre todo dada la finalidad de ofrecer una aproximación a su situación social. Asimismo, este mayor periodo permite realizar de forma adicional cierto análisis de la evolución de la investigación. Además, este periodo se relaciona con los inicios de la democracia y la evolución de las políticas de inclusión en España.

\section{Resultados}

Los resultados obtenidos se exponen de forma independiente ya que obedecen a objetivos distintos, aunque complementarios.

\section{Marco europeo del estudio de las comunidades gitanas}

Comenzaremos este apartado con la revisión sistemática de las investigaciones en referencia al marco europeo.

\section{Análisis dimensional}

En relación a las dimensiones de la exclusión social dentro de los estudios seleccionados, debemos indicar que las cuatro primeras están a mucha distancia del resto. De estas, la primera está referida a participación con un 43\%, la siguiente dimensión es la educación $13 \%$, las investigaciones abordan principalmente los niveles educativos de este grupo, además de que también hacen incidencia en las diferentes que los escolares tienen respecto a otros grupos. Con una menor puntuación quedan los estudios sobre el trabajo (5\%), la salud (3\%) y la vivienda (2\%).

Por último, se sitúan con un $1 \%$ aquellas publicaciones referidas a análisis cultural, consumo, movilidad y marginalidad. No se han encontrado referencias a familia e ingresos, no obstante, son aspectos se han encontrado en relación con análisis cultural-familia, consumo- 
ingresos, movilidad-vivienda, marginalidad-empleo. Debemos realizar un apunte ya que no se cumple del todo la correspondencia con las siete dimensiones, ya que en segundo lugar el objeto de estudio más investigado es la identidad (18\%) visión multidimensional que se relaciona con múltiples ámbitos de la exclusión.

Áreas de estudio que abordan esta temática

En cuanto a las disciplinas que estudian a la población gitana vemos que la sociología es la más prolífera con un $43 \%$, seguida de la antropología $10 \%$, las ciencias políticas $9 \%$, las ciencias de la salud $8 \%$, educación $8 \%$ y a más distancia, la política social y el derecho con un $4 \%$ cada una, la economía $3 \%$, y en la cola la psicología $2 \%$ y el trabajo social $1 \%$. A esta relación se le suma ciencias sociales con un $8 \%$ que combina una perspectiva multidisciplinar.

\section{Contexto geográfico del análisis de las comunidades gitanas en Europa}

Respecto al contexto geográfico en los que se aplica el estudio de las comunidades gitanas queda del siguiente modo. En primer lugar, se sitúa la Unión Europea (36\%), este es el ámbito geográfico en el que mayor número de investigaciones se realiza respecto al colectivo gitano. Se entiende que como unidad supranacional debe regular los flujos migratorios y velar por el bienestar de sus ciudadanos, muchos de estos estudios así lo manifiestan. En segundo lugar, se encuentra el Reino Unido (20\%), seguido de Europa del Este (8\%) como zona geográfica y entre nacional supra-cultural, ya que en esta región de Europa se concentran la mayoría de comunidades gitanas. En el grupo de valores medios están Italia (6\%), España (5\%) y Eslovaquia, Hungría y Rumanía con un 4\% cada una, seguidas de Bulgaria (3\%) y Bélgica (2\%). A la cola de esta distribución estarían una serie de países que en total no llegan al $8 \%$ de la distribución.

\section{Aproximación metodológica al estudio de las comunidades gitanas en Europa}

Acerca del enfoque metodológico debemos de apuntar que casi tres cuartas partes de los estudios $(74,4 \%)$ que se realizan sobre el colectivo gitano usan técnicas cualitativas para la recogida de datos y el análisis. De estas, las entrevistas en profundidad y los grupos de discusión son las más utilizadas. Por el contrario, un 15,4\% optan por metodologías cuantitativas. El análisis estadístico de fuentes secundarias, y en menor medida, las encuestas son las técnicas cuantitativas más usadas. Al igual que en España, la imposibilidad de obtener datos censales sobre esta población hace muy difícil el estudio cuantitativo de las comunidades gitanas. Por último, solo un 10,3\% de las investigaciones recogidas optan por un análisis multimétodo.

\section{Clases de publicación en relación a los diferentes estudios}

En último lugar, tenemos el tipo de publicación que predomina según los procesos de indexación es el artículo científico (90\%) frente al libro (10\%) el que se impone. Así como en los artículos predominan los ámbitos específicos. Sin embargo, en lo que respecta a los libros predominan aspectos más generales que incluyen a la población gitana de modo tangencial (marginalidad, racismo, política, migraciones, etc.)

\section{Estudios sobre la situación social de las comunidades gitanas en España}

En este apartado expondremos los resultados de la revisión exhaustiva llevada a cabo para la investigación de la situación social de las comunidades gitanas en España. 


\section{Estudios según tipos de publicaciones}

Como primer resultado tenemos que a raíz de la extensa revisión bibliográfica realizada se constata la escasez de estudios referentes a este colectivo, ya que solo han sido analizados 62 documentos, entre los que priman los estudios monográficos en formato de libro (23), ya sean informes o estudios, seguidos de los capítulos de libro (18), los artículos (12) y las tesis doctorales (7); en menor medida están las comunicaciones (2).

\section{Investigaciones sobre la situación social de las comunidades gitanas según dimensiones}

En cuanto al estudio de la situación social y la cobertura de las siete dimensiones de la exclusión, son solo siete los informes o estudios que las abarcan, destacando los trabajos coordinados por Laparra (2007 y 2011) que aportan mayor profundidad, junto a otros recientes de ámbito regional, ya sea en Murcia (Vilar, Vilar y Caselles, 2009) o en Asturias (Álvarez, González y San Fabián, 2011), en este grupo estarían otros estudios anteriores y con menor profundidad (Bernal, 1989; González, 1996). Por otro lado, cinco textos abordan varias dimensiones de la exclusión (Calvo, 1989; Arenas, 2012; García, Flecha y García, 2015; Carrón, 2015; Arza y Damonti, 2015 y Leblond, 2018), siendo en todos ellos incluida la educación junto a una o más dimensiones.

Respecto a los estudios con enfoque unidimensional, destacan con clara distancia los que analizan la educación (16), siendo escasos en el resto de dimensiones: vivienda (6), participación (5), salud (4), familia (3), ingresos (3) y empleo (2). Este grupo de estudios, junto a los anteriores multidimensionales serán la fuente de análisis para conocer la situación social del colectivo.

No obstante, se han localizado once documentos, siete de ellos en formato de libro o estudio (Ramírez, 1985; Ardèvol, 1986; Jordan, 1991; Gómez, 1993; Garreta, 2003; Martínez, 2014; Ramírez y Fábregas, 2016;), dos en artículo (Rizo, 2005 y Peñafiel, 2009), una tesis doctoral (Urbiola, 2016) y un capítulo de libro (Laparra, 2011), en los que el análisis se centra en los aspectos culturales e históricos del colectivo gitano, pudiéndose considerar en cierta medida estudios multidimensionales, si bien su objeto de estudio no es tanto la situación social por ámbitos, sino los aspectos de identidad de este colectivo.

\section{Vigencia y cantidad de los estudios realizados}

Otra de las características que priman en estos estudios es su antigüedad, pues la mayoría de bibliografía disponible es anterior a 2010. Perteneciendo la mayoría a la década de los 80. Resulta casi imposible encontrar investigaciones recientes que ofrezcan datos actualizados de la situación social de la población gitana en España. Este fenómeno puede deberse en parte a la dificultad de analizar a la población gitana a través de datos estadísticos, ya que solo se cuenta con estimaciones que varían dependiendo de la fuente. Sin embargo, autores como Carrasco (2004) opinan que se aparenta un gran interés por el estudio de la situación del pueblo gitano y una gran inversión en programas de inclusión, cuando la realidad muestra lo contrario.

En cuanto a las causas del bajo número de estudios existe la creencia de que la dificultas de investigar a la comunidad gitana reside en ella misma, que como mecanismo de defensa se auto-marginan y se trata de un colectivo de difícil acceso. No obstante, Caselles (2013) resalta la necesidad de cambiar la forma de investigar, debiéndose incluir en estos procesos a miembros de la comunidad gitana para poder alcanzar una visión más completa y real de 
su situación social. Así, la realidad muestra que aquellos programas o estudios en los que participan personas pertenecientes al colectivo, o surgen de las propias asociaciones, tienen más éxito que las externas, ya que estas pueden tender a ser menos respetuosas con la cultura gitana (Vilar, Vilar y Caselles, 2009).

Principales retos a los que se enfrentan los estudios sobre comunidades gitanas en España

Una de las principales dificultades a la hora de abordar la investigación de la población gitana en España es la estimación del volumen de población. Existiendo diversidad de aproximaciones. Por un lado, Vilar, Vilar y Caselles (2009) estiman que en España residen entre 650.000 y 1.000.000, siendo el segundo país con más población gitana por detrás de Rumanía. Por otro, Laparra (2014) calcula entre 800.000 y 1,5 millones. Respecto a las autonomías con mayor presencia, serían Andalucía, Cataluña, Madrid y Valencia (Fundación Secretariado Gitano, 2016), mientras en la Región de Murcia se estima una cifra cercana a las 20.000 personas (Vilar, Vilar y Caselles, 2009).

En cuanto a la estructura demográfica, según Laparra y Del Pozo (2007), es una población mayoritariamente joven, siendo las franjas de edad con mayor porcentaje las de 10-19 años, seguida de 30-44 años; encontrando que el número de varones gitanos es superior al de las mujeres gitanas, especialmente en las edades más avanzadas.

En cuanto a su situación social global, según la Fundación FOESSA (2014) el porcentaje de población gitana afectada por la exclusión social es de 72,3\% frente al 23,5\% de la población general.

\section{Análisis dimensional de las principales investigaciones}

Después de hacer una revisión sobre las dificultades que presenta una aproximación demográfica a las comunidades gitanas, pasaremos a describir la situación social de este colectivo, basándonos en los datos extraídos de los diferentes trabajos realizados en España y la realidad ante la exclusión social a nivel multidimensional.

Como se ha dicho, encontramos que son pocos los estudios que realizan un análisis multidimensional de la exclusión social en referencia a este colectivo, la mayoría se centra en uno o dos ámbitos, primando sobre los demás el educativo.

\section{Educación}

La educación conforma una de las herramientas que facilita la inclusión social. La dificultad de la obtención de datos estadísticos, por las dificultades mencionadas en apartados anteriores evita que podamos establecer una imagen global de la situación de las comunidades gitanas ante esta dimensión. Por esta razón, desconocemos el nivel educativo de los miembros de las comunidades, las cifras sobre fracaso/éxito académico, sus tasas de abandono escolar o el número de alumnos que se encuentran escolarizados. Todas las cifras disponibles se elaboran sobre estimaciones y contienen sesgos considerables, debido a estas características debemos tener mucha cautela a la hora de su análisis.

No obstante, este ámbito es el que mayor volumen de texto acumula, tanto junto a otros ámbitos como en solitario, siendo siete de ellos libros o estudios (Calvo, 1989; Fundación Secretariado Gitano, 2013; García, 2017; Iniesta, 1981; Liegeois, 1998; Padilla y Soria, 2015), seis artículos (Parra, Álvarez y Gamella, 1990; Abajo, 1999; Abajo, 2002; Lasa, 2002; 
y Martínez y Alfageme, 2004), una tesis doctoral (Sanchez-Muros, 1990) y dos capítulos de libro (Cardiel, 2007 y López, 2011).

Las investigaciones analizadas arrojan una conclusión generalizada: entre el grupo de escolares el alumnado gitano se encuentra una mayor tasa de absentismo escolar. Esto puede deberse, en parte, a la funcionalidad/utilidad que las familias gitanas otorgan a la educación formal. Esta falta de valoración influye en gran medida en la participación de los padres en el proceso de educación de sus hijos, pieza clave para la continuidad y el éxito escolar (Martínez y Alfageme, 2004). El bajo nivel educativo de las familias condiciona este compromiso, ya que entre la población gitana de mayor edad se dan altas tasas de analfabetismo y, la mayoría de esta población, un menor nivel de estudios.

Diversos autores señalan que los principales factores que influyen en la continuidad educativa y el éxito escolar del alumnado gitano son el cultural, el socioeconómico y el institucional. Aunque es claro que las tres causas están íntimamente relacionadas, Martínez y Alfageme (2004), centran las dificultades educativas en la cuestión socioeconómica, pues la vinculan con los procesos de exclusión social a la que se ha visto sometida históricamente el colectivo, y no tanto a su cultura o modo de vida en sí. Asimismo, añaden que la actitud del profesorado y su conocimiento sobre la cultura gitana resulta un factor determinante a la hora de mejorar el rendimiento del alumnado (Martínez y Alfageme, 2004). Un mayor conocimiento de la realidad social por parte de los maestros podría conseguir que el alumnado se sienta identificado dentro del aula y considere la escuela como un lugar propio mejorando su rendimiento académico y evitando el absentismo escolar. Como señala Carrasco (2004) se debe de tener en cuenta cómo afronta la escuela esta situación y qué puede ofrecer en cuanto a las expectativas reales de este colectivo.

\section{Vivienda}

El ámbito residencial es la segunda dimensión más analizada, incluida en varios estudios multidimensionales y en seis específicos, estos últimos bastante recientes y predominando en estudios globales como capítulos (Campos, 2007; Carrón y Arza, 2013; García, 2011; y Caselles, 2013), tesis doctoral (Nogués, 2010) y el estudio de la Fundación Secretariado Gitano (2015) donde se aporta un reciente mapa sobre vivienda y pueblo gitano en España.

La vivienda configura una de las dimensiones de la exclusión social. Poder disponer de una vivienda estable que cumpla con las condiciones mínimas de habitabilidad condiciona otras dimensiones como la salud y permite un desarrollo positivo para el individuo. Es por ello que, como expresa Campos (2007), la vivienda siempre ha sido uno de los principales ejes de los programas de integración social, especialmente en aquellos destinados a la comunidad gitana.

Históricamente el pueblo gitano ha tenido una tradición nómada en lo que respecta al aspecto residencial. De ahí que se haya visto mermado el derecho a una vivienda adecuada. Cabe destacar que en las décadas de los 70 y 80 del siglo XX, entre un amplio número de comunidades gitanas predominaba el chabolismo, un problema que a pesar de haberse reducido sigue vigente en la actualidad. Sin embargo, como apunta Caselles (2013) prácticamente la totalidad de las infraviviendas (95\%) que existen actualmente en nuestro país siguen habitadas por familias gitanas.

Por otra parte, si nos referimos a las formas de acceso a la vivienda y al tipo de vivienda la Fundación Secretariado Gitano en su reciente estudio (2016) aporta las siguientes 
condiciones, bastante precarias. En cuanto al acceso, para gran parte de las familias de etnia gitana la vivienda protegida acapara más de la mitad de los casos $(54,5 \%)$, seguida de la vivienda libre $(36,0 \%)$ y, en último lugar, están otras formas de acceso a menor distancia de las anteriores $(9,5 \%)$. En relación a la segregación residencial, se observa que esta se compone principalmente de infraviviendas o viviendas en edificios destinados a uso no residencial $(53,4 \%)$, le siguen aquellas viviendas unifamiliares $(29,3 \%) \mathrm{y}$, en menor medida edificios en altura $(17,3 \%)$.

Igualmente, estudios anteriores ponen en evidencia que cada vez es mayor el deterioro de los barrios construidos en décadas anteriores para programas de vivienda para el colectivo gitano, lo que aumenta el hacinamiento y la consolidación de guetos (Campos, 2007).

A lo anteriormente expuesto cabe sumarle que este colectivo se enfrenta a otro gran hándicap en cuanto al acceso al mercado libre de viviendas. Este no es otro sino la discriminación étnica que dificulta adquirir una vivienda en propiedad o acceder a un contrato de alquiler. En los casos en los que se consigue el acceso a la vivienda destaca la falta de acompañamiento social necesario para completar el proceso de integración social (Caselles, 2013).

\section{Red socio-familiar}

La familia es uno de los pilares fundamentales de la comunidad gitana, aun así, son escasos los estudios que se centran la investigación de las redes socio-familiares de apoyo, habiéndose localizado solamente tres que lo analizan de forma independiente (García, 2007; Iturbide, 2011; y Asensio y Lacalzada, 2015).

El pueblo gitano se caracteriza, tradicionalmente, por adoptar el modelo de familia extensa, generalmente numerosa (García, 2007) e interactuar a través de lazos fuertes, lo que hace muy compleja su diversificación de redes sociales fuera del contexto familiar, lo que desencadena que estas comunidades tengan un cierre normativo muy férreo. Por este motivo, suele haber una superposición de ambas estructuras (redes socio-familiares y redes sociales). Debido a esta complejidad, entre las investigaciones analizadas, no hay referencia a estudios dedicados a las redes sociales del pueblo gitano en general, y a las redes socio-familiares en particular. El estudio de este ámbito de la exclusión social no solo es un elemento clave para la inclusión, sino que también supone un reto científico ya que se detecta un espacio de investigación sin explorar, habida cuenta del papel determinante en los procesos de inclusión social.

\section{Empleo}

Un primer análisis sobre comunidad gitana y su situación ante el empleo podría partir de la Encuesta de Población Activa (Instituto Nacional de Estadística). Sin embargo, la legislación vigente impide la clasificación por origen étnico lo que hace imposible acceder a cifras oficiales sobre el pueblo gitano en relación al mercado de trabajo.

La actividad laboral ha sido incluida en varios estudios multidimensionales, si bien solamente como ámbito central en un capítulo de estudios monográficos sobre el colectivo gitano (Laparra y Del Pozo, 2007 y Sánchez y Fernández, 2011).

Laparra y del Pozo (2007) destacan que las tasas de ocupación en la población gitana pueden resultar confusas al compararlas con las tasas generales de población, ya que ambos porcentajes están equilibrados. No obstante, la población gitana experimenta una mayor temporalidad en el empleo. Al mismo tiempo, la escasa formalización de los contratos impide 
la normalización de sus relaciones laborales. A estos dos elementos cabe sumarle el predominio del empleo autónomo, el empleo familiar y el subempleo. Estas circunstancias provocan que solamente algo más de la mitad de la población gitana activa tenga un empleo asalariado. Finalmente, los perfiles laborales de trabajadores gitanos se encuentran caracterizados por su baja formación. Además de por una ocupación en el sector servicios y la venta ambulante.

\section{Ingresos}

En relación con lo expuesto anteriormente, la difícil situación laboral de los miembros de las comunidades gitanas $\mathrm{y}$, por ende, su nivel de ingresos dificulta el acceso a unas óptimas condiciones de bienestar. Por este motivo, de los hogares que padecen pobreza extrema un $75,5 \%$ pertenecen a familias gitanas. Entre aquellos que se encuentran bajo el umbral de la pobreza severa su proporción es de un 26,6\% (Laparra, 2014). En relación a los niveles de pobreza entra la comunidad gitana Legal (2007) hace referencia a la persistencia de la pobreza crónica debido a la prolongación de las condiciones de precariedad en el tiempo.

No obstante, al igual que ocurre en otros ámbitos, nos encontramos con dificultades a la hora de evaluar la estimación de los ingresos entre la población gitana (Legal, 2007), debido a la ausencia de datos oficiales.

Sin duda, la precariedad económica a la que un gran parte de la población gitana se enfrenta les lleva a buscar la complementariedad de ingresos apoyándose en la familia como unidad económica, en la que todos los miembros aportan según su capacidad. En este sentido, la dimensión ingresos se encuentra íntimamente relacionada con la dimensión red sociofamiliar a través de la solidaridad económica.

\section{Salud}

A continuación, abordamos la dimensión salud, de la cual se han localizado cuatro estudios, dos en forma de capítulo de libro en estudios monográficos (Campos y Laparra, 2007 y Arza, 2011) y otros dos como artículos (Ferrer, 2003 y Cruz, 2015).

Tenemos que ser conscientes de que esta dimensión es múltiple, ya que engloba factores biopsico-sociales y ambientales, no solamente cuenta el buen estado físico o la ausencia de enfermedad, sino también aspectos psicológicos y cómo influye nuestro entorno (socioeconómico, familiar, laboral, medioambiental, etc.), tal como define la OMS (2013).

Las dificultades para recabar datos precisos sobre la población gitana son enormes, debido a que los registros sanitarios tienen una especial protección al considerarse altamente sensibles. Como señala Ferrer (2003) a pesar de que los diversos estudios señalan que los miembros de las comunidades gitanas en España poseen una salud vulnerable y afrontan grandes desigualdades en este ámbito, son relativamente escasos los trabajos realizados al respecto. Por otra parte, los estudios existentes suelen ser de carácter local y se centran en aspectos médicos, por lo que no tienen en cuenta la percepción que sobre esta dimensión posee este colectivo (Arza, 2011). Así, en el análisis de Arza (2011) sobre las condiciones de salud de este grupo se destacan el alto nivel de obesidad y tabaquismo, además de un mayor número de diagnósticos de enfermedades crónicas en comparación con el total de la población. Este mayor porcentaje de afecciones o de consultas médicas con respecto a la población general está directamente relacionado con la situación económica y las condiciones de vivienda a las 
que el colectivo se enfrenta. También destaca que acuden más a consulta utilizan más el servicio de urgencias.

Los hábitos de consumo repercuten en la salud de un modo considerable, muchas patologías devienen de prácticas poco saludables, que en definitiva son patrones culturales. En las sociedades industrializadas es común que la población con menos recursos consuma alimentos hipercalóricos y procesados, lo que provoca altos niveles de riesgo en patologías cardiovasculares.

\section{Participación social}

Como última dimensión de análisis trataremos la participación social, la cual ha sido objeto de estudio en cinco documentos analizados: dos artículos (Aguilera, 1988 y Laparra, 2005), en un capítulo de libro monográfico (Cartiel y Laparra, 2007) y en una reciente tesis doctoral (Peña, 2017).

Entre las comunidades gitanas, como destacan Cardiel y Laparra (2007), aquellas iniciativas de participación social que más éxito tienen son aquellas que nacen del propio colectivo o en las participan miembros de la comunidad. También, es frecuente que en muchos casos haya una instrumentalización de la participación social y una tutorización de su participación por parte de terceros. A la hora de participar, suelen involucrarse en mayor medida en asociaciones de carácter religioso. Del mismo modo, se observa que la participación aumenta con el nivel educativo y económico. En este sentido, Laparra (2011) argumenta que se trata de un colectivo sobrerrepresentado en la exclusión social, lo que tiene un efecto directo en su participación social, cuyo déficit es más cualitativo que cuantitativo ya que, aunque si participan en asociaciones lo hacen en aquellas con menos influencia social.

\section{A modo de síntesis}

A nivel internacional la revisión sistemática puede conducir, en un principio, a equívocos. Ya que al introducir los términos de búsqueda en las bases de datos obtenemos un considerable volumen de documentos. Sin embargo, una vez depurados los estudios en función de su temática, los resultados muestran una significativa escasez de investigaciones que traten la situación social de las comunidades gitanas. Una abrumadora mayoría de las investigaciones son artículos que se centran en exclusiva en alguno de los aspectos de la exclusión. Asimismo, se observan grandes dificultades a la hora de acceder a cifras reales. De ahí que los análisis cuantitativos no lleguen a ser ni siquiera una quinta parte del total. Por otro lado, entre las dimensiones de la exclusión, la participación y la educación abarcan casi la mitad de los estudios realizados sobre comunidad gitana y exclusión social.

La identidad es otra área de estudio muy investigada, siendo de carácter transversal ya que en sentido amplio abarca diversas dimensiones de la exclusión social, abordando el liderazgo, la autogestión o la diferencia de roles entre hombres y mujeres.

Según la distribución geográfica de los estudios, se aprecia que la Unión Europea, el Reino Unido y Europa del Este son los lugares que concentran casi dos tercios de las investigaciones sobre las comunidades gitanas. Llama la atención que fuera del continente europeo no haya apenas referencias a esta comunidad. En último lugar, entre las disciplinas de estudio que investigan la exclusión social destaca la sociología que abarca casi la mitad de los trabajos 
analizados, seguida de la antropología, la ciencia política, la salud y la educación. A más distancia, la economía, el derecho, la psicología y, por último, el trabajo social.

Cuando abordamos el análisis exhaustivo en España sobre la exclusión social de las comunidades gitanas, la primera conclusión que sacamos es que los estudios son escasos. Además, la mayoría de estudios disponibles no están actualizados, lo que impide analizar la realidad actual de la población gitana en España. Muchas de las investigaciones tienen buena calidad, sin embargo, es imposible hacer un seguimiento longitudinal de la situación de las comunidades gitanas porque no existe una continuidad en las diferentes líneas de investigación.

Si a esto le sumamos la indefinición en la denominación de esta etnia, entonces debemos tener en cuenta que no existe un colectivo gitano, sino múltiple y diversas: comunidades gitanas. A esto se le añade que, a causa de la legislación vigente, y con el ánimo de evitar la estigmatización social de cualquier grupo étnico, oficialmente no se pueden realizar censos que desagreguen a la población por origen identitario-cultural. Este hecho tiene dos consecuencias, por una parte, inhibe la realización de investigaciones sobre esta población. Y por otra, esta consecuencia tiene que ver con el perfil metodológico de los estudios, en este caso observamos que las investigaciones de carácter cualitativo priman sobre las que aplican un enfoque cuantitativo.

Aunque se han localizado doce estudios con carácter multidimensional, solo dos de ellos abordan todas las dimensiones de la exclusión y con un análisis pormenorizado. El resto trata varias de las dimensiones de forma bastante transversal.

Respecto a las dimensiones más analizadas de forma individual, destaca con creces la de la formación/educación, dando cuenta de uno de los principales problemas que afecta a este colectivo, o al menos del interés científico que esta dimensión suscita. El resto de dimensiones tienen menor protagonismo y suelen analizarse vinculadas a otras (empleoingresos; familia-participación; red sociofamiliar-participación, etc.).

En contraste con el análisis realizado sobre las investigaciones a nivel europeo se aprecia que no existe una misma tendencia en función del estudio de los diferentes ámbitos de la exclusión, primando en Europa aquellas investigaciones sobre la participación y la identidad, estos últimos con marcado carácter multidimensional, mientras que en España se acentúa el interés por los aspectos básicos de integración social, como la educación.

\section{A modo de discusión}

En la actualidad, el colectivo gitano sigue sin estar plenamente integrado en nuestra sociedad. Persisten una serie de barreras estructurales que impiden la plena participación en la vida política y social. No aportamos nada nuevo al decir que la comunidad gitana ha sido y continúa siendo, en gran medida, una de las grandes afectadas por la exclusión social a lo largo de la historia, no solo en España, sino en toda Europa. Sin embargo, las comunidades gitanas poseen características diferentes dependiendo del lugar o país en el que se asienten. Por este motivo, tratar al pueblo gitano como una unidad homogénea y uniforme introduciría un sesgo difícilmente salvable. Desde este punto de vista, aunque puedan llegar a parecer sinónimos, parece más razonable denominar pueblo gitano al grupo étnico y como comunidades gitanas a los grupos humanos de esta etnia que conviven en un mismo territorio. 
Como hemos comprobado o a lo largo de este trabajo existe una dificultad manifiesta a la hora de encontrar datos fiables y actuales sobre el colectivo gitano. Esto determina el poder poseer una comprensión actual sobre la situación social de esta población. Aspectos éticoslegales relacionados con la protección contra la discriminación no permiten la elaboración de censos que recojan la estructura demográfica, la composición de los hogares, su ubicación y la cuantificación de estas comunidades. Consecuencia de esto es que a día de hoy no se tengan cifras aproximadas sobre el número de personas que pertenecen a este grupo. Lo que impide que las políticas orientadas a su inclusión social puedan tener un diseño adecuado al colectivo y a su participación. Se precisa, por tanto, de un enfoque multidimensional puesto que la exclusión es un proceso dinámico, que tenga como resultado una política integral y holística, ya que todos sus ámbitos estás relacionados entre sí.

Por otra parte, el grueso de las investigaciones encontradas aborda en profundidad la situación social del colectivo desde una perspectiva unidimensional, lo que impide disponer de una visión de conjunto sobre los problemas a los que estas comunidades se enfrentan. A esto se le suma que se encuentran dificultades teórico-empírico-operativas para poder seguir el modelo multidimensional de la exclusión social, ya que es complejo de ubicar algunos estudios. La falta de continuidad y la disparidad de propuestas provocan que no sea fácil aunar distintas dimensiones en un único modelo de análisis. Un reto que debe solucionar la comunidad científica a través de nuevos trabajos que integren las dimensiones de la exclusión conjuntamente a dimensiones transversales como la identidad y la cultura. Todo esto, sin despreciar enfoques profundos de ámbitos específicos.

Al mismo tiempo, los estudios tienen un marcado sesgo institucional, el tratamiento de las comunidades gitanas es casi siempre ajeno a la visión que el propio colectivo tiene de sí mismo. Por esta razón, se evidencia la necesidad de hacer estudios multidimensionales de corte cuantitativo y cualitativo que den voz la población gitana.

Este trabajo es una primera aproximación al análisis de las diferentes investigaciones sobre la exclusión social del colectivo gitano. Por tanto, somos conscientes de que el proceso de selección y acercamiento a estos estudios contiene ciertos sesgos metodológicos, que van desde los criterios de búsqueda y selección, hasta los de análisis de los documentos. Por ese motivo, se estima conveniente avanzar en este tipo de aproximaciones, ahondando aún más en la precisión de la estrategia metodológica y por tanto, en su calidad científica. En particular es importante, mejorar la calidad y cantidad de la muestra, aumentando las bases de datos consultadas, evitando las redundancias y acotando más los criterios de búsqueda.

Por último, queda constancia de la necesidad de realizar nuevos estudios, cambiando la mirada e intentado dar una visión global y realista desde una perspectiva multidimensional de la situación de la comunidad gitana. Y sobre todo, haciendo copartícipes de una manera activa en el estudio de su propia situación social a las personas de esta etnia. Solamente mediante la implementación de metodologías de análisis en las que quepa el papel activo de este colectivo, investigación-acción, será posible realmente conocer su situación social, y por ende, poder establecer prioridades que contribuyan al diseño de políticas de intervención verdaderamente eficaces para promover la inclusión social. 


\section{Referencias}

Abajo, J. E. (1999). La situación de los niños gitanos en España: Un test a nuestro sistema social y escolar. Revista Interuniversitaria de Formación del profesorado (36), pp. 57-99.

Abajo, J. E. (2002). Cultura gitana y escuela. Arakerando (86), pp. 8-16.

Aguilera Cortes, P. (1988). La participación política en la comunidad gitana. Gitano Gitanos: Pensamiento y Cultura. Revista Bimestral de la Fundación Secretariado (4), pp. 16-19.

Álvarez, M. V.; González, M. M. y San Fabián, J. L. (2011). La situación de la infancia gitana en Asturias. Oviedo: Consejería de Bienestar Social y Vivienda. Gobierno del Principado de Asturias. Recuperado de http://cort.as/-HfKp consultado el 01/10/2018.

Ardèvol, E. (1986). Vivencias y cambio en la cultura de los gitanos. Madrid: Alianza Editorial.

Arenas Martínez, M. (2012). Bases de análisis e intervención con la minoría gitana. Cuadernos de Trabajo Social, 25(1), pp. 103-112.

Arza, J. (2011). La salud en la comunidad gitana: desigualdad acentuada por el género, la edad y la exclusión. En M. Laparra, (coord.) Diagnóstico social de la comunidad gitana en España. Un análisis contrastado de la Encuesta del CIS a Hogares de Población Gitana 2007. Madrid: Ministerio de Sanidad, Política Social e Igualdad.

Arza, J. y Damonti, P. (Coord.). (2015). Exclusión en la comunidad gitana. Una brecha social que persiste y se agrava. En F. Lorenzo, VII Informe sobre exclusión y desarrollo social en España. Madrid: Fundación FOESSA

Asensio, A. y Lacalzada, M. J. (2015). Mujeres gitanas de Zaragoza: de lo privado a lo público, un análisis desde la perspectiva de género (Tesis doctoral). Universidad de Zaragoza.

Bernal Palacios, J. M. (1989). Los gitanos en la región de Murcia. Murcia: Concejalía de servicios sociales. Murcia: Ayuntamiento de Cartagena.

Calvo Buezas, T. (1989a). Los racistas son otros: gitanos, minorías y derechos humanos en los textos escolares. Madrid: Editorial Popular.

Calvo Buezas., T. (1989b). ¿España racista? Voces payas sobre los gitanos. Barcelona: Anthropos Editorial de Hombre.

Cardiel, B. (2007). Educación y comunidad gitana. En M. Laparra (coord.), Situación social y tendencias de cambio en la comunidad gitana. Pamplona: Universidad Pública de Navarra-

Campos, B. (2007a). La salud en comunidad gitana. En M. Laparra, Situación social y tendencias de cambio en la comunidad gitana. Pamplona: Universidad Pública de Navarra-

Campos, B. (2007b). La vivienda en la comunidad gitana. En M. Laparra. Situación social y tendencias de cambio en la comunidad gitana. Pamplona: Universidad Pública de Navarra- 
Cardiel, B y Laparra, M. (2007). Participación social y política de la comunidad gitana. En Laparra, M. (coord.). Situación social y tendencias de cambio en la comunidad gitana. (pp. 171-191). Pamplona: Universidad Pública de Navarra.

Carrasco, S. (2004). Experiencias y trayectorias de éxito escolar de gitanas y gitanos en España. Madrid: Centro de Investigación y Documentación Educativa. Recuperado de http://cort.as/-HfJa consultado el 04/10/2018.

Carrón, J. (2015). La situación demográfica y social de la comunidad gitana. En Jornadas sobre Abordaje Integral de la Comunidad Gitana en las consultas de Atención Primaria. Zaragoza: Servicio Aragonés de la Salud y Dirección General de Salud Pública.

Carrón, J. y Arza, J. (2013). Intensificando (aún más) la vulnerabilidad: los hogares monoparentales en la población gitana. En S. Giménez Rodríguez y G. Tardivo (Coords.). Proyectos sociales, creativos y sostenibles (pp. 66-80). Toledo: ACMS,

Caselles, J. F. (2013). Vivienda y pueblo gitano. En M. Hernández Pedreño Vivienda y exclusión residencial (pp. 269-288). Murcia: Editum.

Cruz, J. M. (2015). Importancia de los agentes de salud dentro de la etnia gitana y actuación de enfermería. Revista Enfermería CyL, 7(2), pp. 69-88.

Fernández, A. B. y García, O. (2018). Situación social en ingresos, vivienda y salud. En Hernández Pedreño, M. (Coord.) Inserción Social y laboral de los jóvenes en la Región de Murcia (pp. 203-249). Murcia: Editum

Ferrer, F. (2003). El estado de salud del pueblo gitano en España. Una revisión de la bibliografía. Gaceta sanitaria: Órgano oficial de la Sociedad Española de Salud Pública y Administración Sanitaria, 17(3), pp. 2-8.

Francolí, N. y Camarasa, M. (2012). Informe de investigación proyecto empow-air Estado español. Surt. Fundació de dones. Fundació Privada. Recuperado de http://cort.as/-HfJf consultado el 01/10/2018.

Fundación Secretariado Gitano (2016). Estudio-Mapa sobre Vivienda y Población Gitana, 2015. Resumen Ejecutivo. Septiembre de 2016. Madrid: Ministerio de Sanidad, Servicios Sociales e Igualdad.

Fundación Secretariado Gitano. (2013). El alumnado gitano en secundaria: un estudio comparado. Madrid: Ministerio de Educación, Cultura y Deporte.

García, A. (2007). La familia en la comunidad gitana. En M. Laparra, Situación social y tendencias de cambio en la comunidad gitana. Pamplona: Universidad Pública de Navarra-

García, A. (2011). La reconversión de los problemas de vivienda de la comunidad gitana: de la chabola a una vivienda inadecuada. En M. Laparra, (coord.) Diagnóstico social de la comunidad gitana en España. Un análisis contrastado de la Encuesta del CIS a Hogares de Población Gitana 2007. Madrid: Ministerio de Sanidad, Política Social e Igualdad.

García, J.A. (Coord.) (2017). La cultura del pueblo gitano en el currículo de la educación obligatoria, a través de su presencia, ausencia y percepción en los libros de texto. Madrid: Ministerio de Educación, Cultura y Deporte. 
García, T., Flecha, R., García, F. J. (2015). Impacto político de la investigación europea sobre el pueblo gitano: el caso del plan integral del pueblo gitano en Cataluña. Tesis doctoral. Granada: Universidad de Granada.

Garreta Bachoca, J. (2003). La integración sociocultural de las minorías étnicas (gitanos e inmigrantes). Barcelona: Anthropos editorial.

Gómez Alfaro, A. (1993). La gran redada de gitanos: España, la prisión general de gitanos en 1749. Madrid: Presencia Gitana.

González Barbero, M. A. (1996). Trabajando con gitanos: aproximación al estudio de la realidad del colectivo gitano. Madrid: Cáritas Española.

Hernández Pedreño, M. (2010). El estudio de la pobreza y la exclusión social. Aproximación cuantitativa y cualitativa. Revista interuniversitaria de formación del profesorado (69), pp. 25-46.

Hernández Pedreño, M. (Dir.) (2016). Exclusión residencial en Murcia. Miradas y trayectorias. Murcia: CEPAIM.

Hernández Pedreño, M. (Dir.) (2018). Inserción social y laboral de los jóvenes en la Región de Murcia. Murcia: Editum.

Iniesta, A. (1981). Los Gitanos: Problemas Socioeducativos. Madrid: Narcea.

Iturbide, R. (2011). Lento cambio en la familia gitana. En M. Laparra, (coord.) Diagnóstico social de la comunidad gitana en España. Un análisis contrastado de la Encuesta del CIS a Hogares de Población Gitana 2007. Madrid: Ministerio de Sanidad, Política Social e Igualdad.

Jordan Peman, F. (1991). Religiosidad y moralidad de los gitanos en España. Madrid, España: Asociación Secretariado General Gitano.

Laparra, M. (2005). La Europa de los Gitanos: Identidad, participación y políticas sociales en la Europa ampliada y su incidencia en España. Documentación Social (137), pp. 1535. Recuperado de http://cort.as/-HfKR consultado el 07/10/2018.

Laparra, M. (2011). Diagnóstico social de la comunidad gitana en España. Un análisis contrastado de la Encuesta del CIS a Hogares de Población Gitana 2007. Informes, Estudios e Investigación 2011. Madrid: Ministerio De Sanidad, Política Social E Igualdad.

Laparra, M. (2011). Participación social y dinámica comunitaria de una comunidad excluida. En M. Laparra, (coord.) Diagnóstico social de la comunidad gitana en España. Un análisis contrastado de la Encuesta del CIS a Hogares de Población Gitana 2007. Madrid: Ministerio de Sanidad, Política Social e Igualdad.

Laparra, M. (2014). La fractura social se ensancha: intensificación de los procesos de exclusión en España durante 7 años. En: F. Lorenzo, (Coord.), VII Informe sobre exclusión y desarrollo social en España. Madrid: Fundación FOESSA.

Laparra, M. (coord.) (2011). Diagnóstico social de la comunidad gitana en España. Un análisis contrastado de la Encuesta del CIS a Hogares de Población Gitana 2007. Madrid: Ministerio de Sanidad, Política Social e Igualdad. Recuperado de http://cort.as/HfJu consultado el 06/10/2018. 
Laparra, M. y Del Pozo, J. M. (2007). Empleo y ocupación en la comunidad gitana. En M. Laparra (Coord.), Situación social y tendencias de cambio en la comunidad gitana. Pamplona: Universidad Pública de Navarra.

Laparra, M. y García, A. (2011). Pobreza y privación en la comunidad gitana. En M. Laparra, (coord..), Diagnóstico social de la comunidad gitana en España. Un análisis contrastado de la Encuesta del CIS a Hogares de Población Gitana 2007. Madrid: Ministerio de Sanidad, Política Social e Igualdad.

Laparra, Miguel. (coord.) (2007). Situación social y tendencias de cambio en la comunidad gitana. Pamplona: Universidad Pública de Navarra.

Lasa, B. (2002). La escolarización, una realidad creciente entre las familias gitanas. Aula de Infantil (9), pp. 34-36.

Leblond, B. (2018). Los gitanos de España: el precio y el valor de la diferencia. Madrid: Gedisa.

Legal, I. (2007). Situación económica de la comunidad gitana. En M. Laparra (coord.), Situación social y tendencias de cambio en la comunidad gitana. Pamplona: Universidad Pública de Navarra.

Liégeois, J. P. (1998). Minoría y Escolaridad: El paradigma gitano. Madrid: Editorial Presencia Gitana/Interface.

López, M. (2011). La inserción educativa de la comunidad gitana: ¿realidad o espejismo? En M. Laparra, (coord.) Diagnóstico social de la comunidad gitana en España. Un análisis contrastado de la Encuesta del CIS a Hogares de Población Gitana 2007. Madrid: Ministerio de Sanidad, Política Social e Igualdad.

Martínez, M. (2014). Los gitanos y las gitanas de España a mediados del siglo XVIII: el fracaso de un proyecto de exterminio. Almería: Editorial Universidad de Almería.

Martínez, M. y Alfageme, A. (2004). Integración socioeducativa del Alumnado gitano en la escuela española. Revista Española de Educación Comparada (10), 299-323.

Nogués, L. J. (2010). Exclusión residencial y políticas públicas: el caso de la minoría gitana en Madrid (1986-2006) (Tesis doctoral). Universidad de Granada. Recuperado de http://cort.as/-HfKA consultado el 03/10/2018.

Organización Mundial de la Salud (2013). Informe sobre la salud en el mundo 2013: investigaciones para una cobertura sanitaria universal. Ginebra: OMS.

Padilla, T. y Soria, A. A. (2015). Facilitando el apoyo a los estudiantes gitanos para el acceso a la educación superior: Buenas prácticas para mejorar la participación de la población gitana en la educación superior en España. Informe del proyecto Higher Education Internationalisation and Mobility: Inclusion, Equalities and Innovation Project. Marie Sklodowska-Curie Actions, Research and Innovation Staff Exchange, Horizon 2020. Grant agreement. Recuperado de http://cort.as/-HfKL consultado el 10/10/2018.

Parra, I., Álvarez-Roldán, A, Gamella, J. (2017). Un conflicto silenciado: Procesos de segregación, retraso curricular y abandono escolar de los adolescentes gitanos. Revista de Paz y Conflictos, 10 (1), pp. 35-60. 
Peña, M. P. (2017). El asociacionismo romaní en Europa como nuevo intermediario entre los Estados-naciones, las Instituciones-supra-nacionales y las comunidades gitanas (Tesis Doctoral). Universidad de Granada. Recuperado de http://cort.as/-HfKR consultado el 15/9/2018.

Peñafiel, A. (2009). Los gitanos en España y Región de Murcia: Seis siglos de marginación. Anales de Historia Contemporánea, 25 (2009), pp. 37-56.

Pérez-Herrero, M.H., Antúnez, A. y Burguera Condon, J. (2017). Factores que inciden en el abandono escolar del alumnado gitano: perspectiva de profesionales clave. Revista de Psicología y Educación, 12(1), pp. 55-76.

Ramírez-Heredia, J. D. (1985). En defensa de los míos: ¿qué sabe Vd. de los gitanos?. Barcelona: Ediciones 29.

Ramírez-Heredia, J. D. y Fábregas Hernández, A. (2016). ¿Periodistas contra el racismo? la prensa española ante el pueblo gitano. Barcelona: Centro de Producción Editorial y Divulgación audiovisual (CPEDA).

Rizo, A. E. (2005). Apuntes sobre la Comunidad Gitana Española: Breves Trazos de su Historia en Conexión con el Contexto Europeo. Diálogos Revista Electrónica de Historia, 6(1), pp. 179-229. Recuperado de http://cort.as/-HfKU consultado el 01/10/2018.

Sánchez, B. y Fernández, A. (2011). La situación laboral de la población gitana: conocimiento y reconocimiento. En M. Laparra, (coord.), Diagnóstico social de la comunidad gitana en España. Un análisis contrastado de la Encuesta del CIS a Hogares de Población Gitana 2007. Madrid: Ministerio de Sanidad, Política Social e Igualdad.

Sánchez-Muros Lozano, P. S. (1990). Hablando de los gitanos: Representaciones sociales en el discurso y la interacción escolar (Tesis Doctoral). Universidad de Granada. Recuperado de http://cort.as/-HfKa consultado el 11/10/2018.

Urbiola, A. (2016). El valor de la diversidad: Ideología multicultural y reducción del prejuicio hacia las personas gitanas (Tesis Doctoral). Universidad de Granada.

Vilar, J. B., Vilar, M. J., Caselles, J.F (2009). La comunidad gitana en España y Región de Murcia: entre la integración y la exclusión. Anales de Historia Contemporánea, 25, pp. 25-34. 